\title{
A Case of Transposition of Great Arteries with Ventricular Septal Defect and Mesocardia Presenting for Neurosurgery
}

\author{
Soniya Biswas ${ }^{1,(0)}$ Keta Thakkar ${ }^{1}$ Neeraja Ajayan ${ }^{1}$ \\ ${ }^{1}$ Division of Neuroanesthesia, Department of Anaesthesiology, \\ Sree Chitra Tirunal Institute for Medical Sciences and Technology, \\ Trivandrum, Kerala, India
}

\author{
Ajay Prasad Hrishi ${ }^{1} \quad$ Manikandan Sethuraman ${ }^{1}$
}

\begin{abstract}
Address for correspondence Ajay Prasad Hrishi, DM, Division of Neuroanesthesia, Department of Anaesthesiology, Sree Chitra Tirunal Institute for Medical Sciences and Technology, Trivandrum 695011, Kerala, India (e-mail: drajay@sctimst.ac.in).
\end{abstract}

\author{
Abstract \\ Keywords \\ - neurosurgery \\ - transposition of great \\ arteries \\ - ventricular septal \\ defect
}

Congenitally corrected transposition of great arteries (CC-TGAs) associated with a ventricular septal defect (VSD) presents with cyanotic spells and systemic complications such as brain abscess. In mesocardia, the heart lies in the midline with no apex seen on the left side. We report the anesthetic management of a child with CC-TGA, VSD, and mesocardia presenting with parietal brain abscess for neurosurgery. The significant anesthetic challenges include maintenance of peripheral vascular resistance lower than systemic vascular resistance, prevention of air embolism and paradoxical embolism, avoidance of hyperviscosity in addition to avoiding any rise in intracranial pressure, and maintenance of cerebral perfusion pressure.

\section{Introduction}

Congenitally corrected transposition of great arteries (CC-TGA) is a sporadic disorder, which constitutes 0.5 to $1 \%$ of the total congenital cardiac defects. Mesocardia is a condition in which the heart lies in the midline with no apex seen on the left side. CC-TGA with a ventricular septal defect (VSD) presents with cyanotic spells and can result in systemic complication such as brain abscess. We report the anesthetic management of a child with CC-TGA, VSD, and mesocardia presenting with parietal brain abscess for neurosurgery. Informed consent was obtained from the parent before the submission of this case report.

\section{Case Presentation}

A 5-year-old boy weighing $18 \mathrm{~kg}$ presented to us with moderate, holocranial, nonradiating headache associated with multiple episodes of nausea and vomiting since the past 4 days. He was a known case of CC-TGA and VSD with a history of cyanotic spells from day 3 of life after a term delivery. Computed tomography scan of the head revealed a well-defined hypodense lesion measuring $31 \times 34 \times 26 \mathrm{~mm}$ in the left parietal region with associated perilesional edema in the adjacent frontoparietal white matter ( $\mathbf{- F i g .} \mathbf{1}$ ). On examination, the child was febrile with a heart rate (HR) of 91 beats/min, blood pressure of $102 / 62 \mathrm{~mm} \mathrm{Hg}$, and room air blood oxygen saturation of $92 \%$. On chest auscultation, a loud S1, S2 (P2 > A2) with ejection systolic murmur was appreciated at the pulmonary area. Preoperative blood investigations were within normal limits, and chest X-ray had evidence of mesocardia ( - Fig. 2). Echocardiography confirmed the presence of TGA with large VSD and severe pulmonary valve stenosis (gradient $>50 \mathrm{~mm} \mathrm{Hg}$ ). The child was posted for emergency surgery under general anesthesia for evacuation of the abscess. In the operating room, after attaching standard preinduction monitors consisting of five lead electrocardiogram (ECG), pulse oximetry $\left(\mathrm{SpO}_{2}\right)$, and noninvasive blood pressure, the child was preoxygenated with $100 \%$ oxygen for 5 minutes. Anesthesia was induced with intravenous (IV) ketamine 3 to $5 \mathrm{mg} / \mathrm{kg}$, fentanyl 1 to $2 \mu \mathrm{g} / \mathrm{kg}$, and vecuronium $0.1 \mathrm{mg} / \mathrm{kg}$ was administered after confirming ventilation. The trachea was intubated with 5.0 cuffed endotracheal tube, and an additional large-bore IV access was secured. Precautions were taken to avoid air bubbles in the IV fluid system by using an infusion set with a bubble trap. Infective endocarditis prophylaxis was given 30 minutes before incision. 


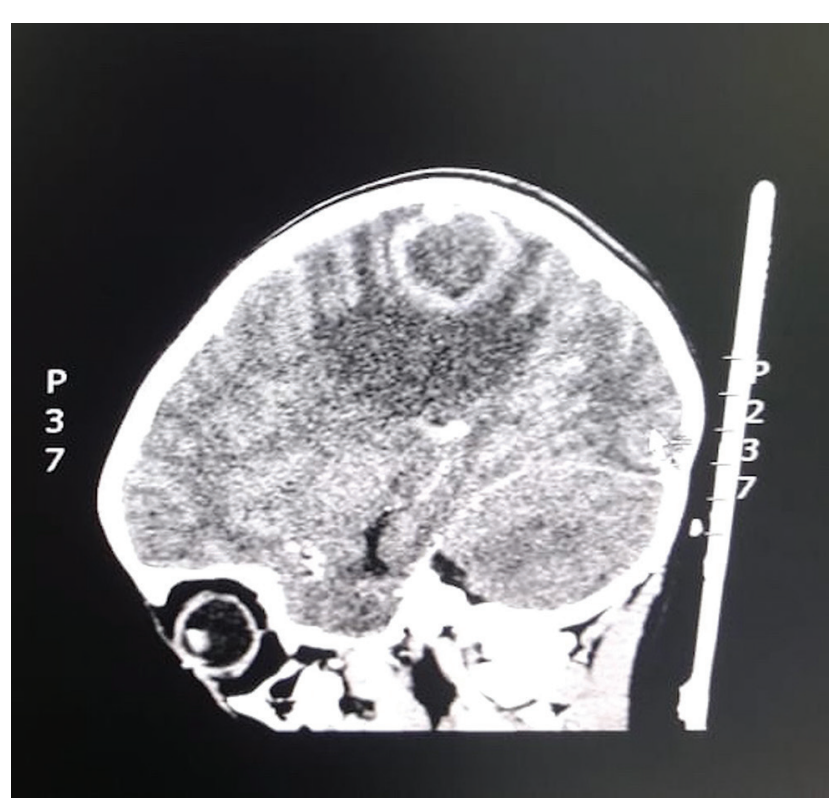

Fig. 1 Computed tomography (sagittal section) of the brain showing a ring enhancing well-defined hypodense lesion in the left frontal region with associated perilesional edema in adjacent frontoparietal white matter.

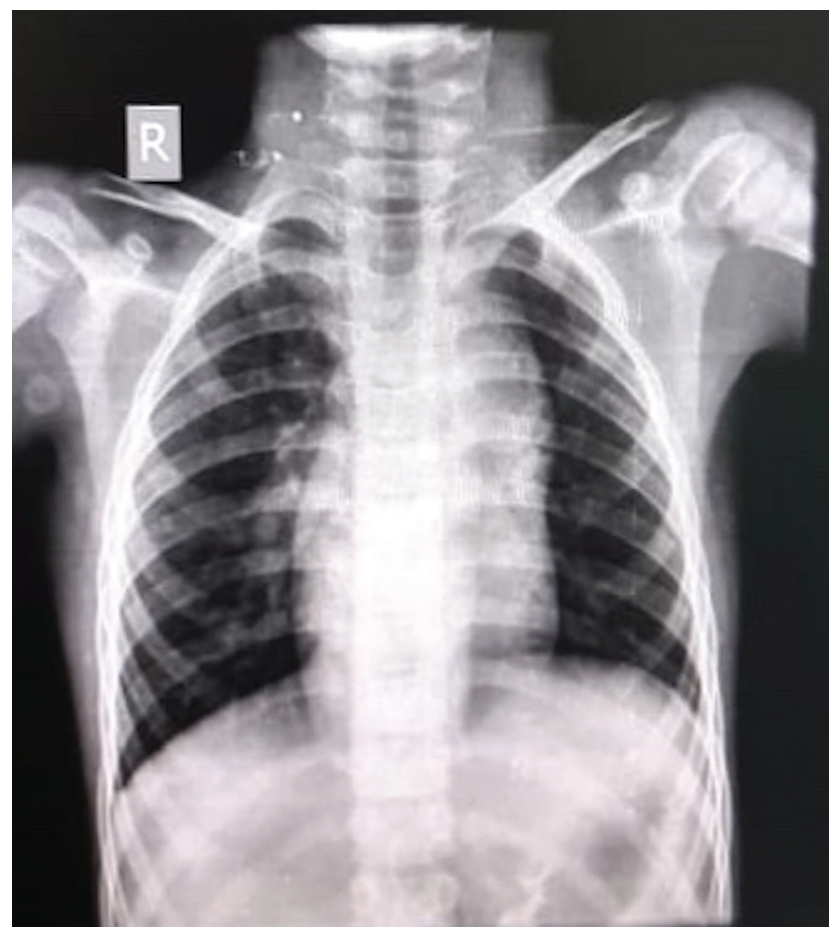

Fig. 2 Chest radiograph showing mesocardia as the longitudinal axis of the heart lies in the mid-sagittal plane and the heart has no apex.

Anesthesia was maintained with 1.5 to $2 \%$ sevoflurane (0.7-0.9 minimum alveolar concentration [MAC]) with air:oxygen mixture of 50:50 and nitrous oxide $\left(\mathrm{N}_{2} \mathrm{O}\right)$ was avoided. Additional monitoring consisted of invasive blood pressure, nasopharyngeal temperature, and capnography (end-tidal $\mathrm{CO}_{2}$ ). Intraoperative cardiac monitoring was done with transesophageal echocardiography (TEE). The mid-esophageal bicaval view was preferred during the burr hole phase to screen for venous air embolism (VAE). IV paracetamol $20 \mathrm{mg} / \mathrm{kg}$ was given for postoperative analgesia. The child was kept normothermic, normocapnic, and euvolemic intraoperatively. Post surgery, the neurological status was assessed and as the child was fully awake and obeying commands, trachea was extubated. In the postoperative period, he remained afebrile, ambulant, and did not develop any neurological deficits.

\section{Discussion}

CC-TGA is a cardiac anomaly in which there is an interchange of the aorta and pulmonary arteries origin and so are the functions of the right and left ventricles (LVs) as well as the mitral and tricuspid valves. In this condition, both ventricles and their attached valves are reversed. The weaker right ventricles (RVs) along with tricuspid valves are on the heart's left side and pump blood through the aorta, and the stronger LV is on the right and pumps blood through the pulmonary arteries. This condition is also called Levo-TGA and is very commonly associated with VSD, atrial septal defect, patent foramen ovale, patent ductus arteriosus, and valvular dysfunction. ${ }^{1}$ Patients with CC-TGA can be asymptomatic or present with an incidental finding of a cardiac murmur, conduction abnormalities, heart failure, or cyanotic spells. When CC-TGA is associated with VSD, cyanosis will be present and can also result in serious complication such as brain abscess. ${ }^{2,3}$ Such cardiogenic brain abscess caused due to hematogenous spread of bacteria usually presents in the initial decade of life. Most common symptoms of brain abscess are headache, vomiting, and fever and can also result in focal neurological signs and very rarely seizures.

Our patient had CC-TGA with a large VSD and severe pulmonary stenosis with a history of cyanotic spells. Anesthetic concerns in our patient were a probable worsening of the RV function resulting in heart failure, pulmonary hypertension, and reversal of shunt. Additional concerns were raised intracranial pressure (ICP), hypovolemia, and dehydration due to fever and vomiting. These patients are prone for right heart failure as the structurally "weaker" RV has to pump blood against high systemic vascular resistance (SVR) and the majority of patients present with congestive heart failure. Thus, a thorough cardiac evaluation is required to assess the biventricular function as this subset of patients are prone for heart failure due to additional stress on the RV by the presence of VSD and LV failure due to pulmonary hypertension. A detailed birth history, history of cyanotic spells, any medical or surgical intervention also needs to be elicited. Hemogram will aid in screening for anemia, erythrocytosis (hyperviscosity syndrome) as these conditions can hamper the tissue oxygen delivery. Coagulation profile needs to be done to detect any hypercoagulable state which is commonly associated with CC-TGA as this presents an increased risk of pulmonary and systemic thromboembolism. Preoperative room air oxygen saturation will give an idea of the systemic oxygenation and functional status of the cardiopulmonary system. ${ }^{1}$ Preoperative chest X-ray will help us to rule out any pulmonary 
infections and pulmonary edema. Baseline ECG may reveal conduction or rhythm abnormalities which are seen in this subset of patients due to unusual atrioventricular (AV) node position or aberrant course of bundle of His. ${ }^{4}$ Atrial dilatation due to $\mathrm{AV}$ regurgitation also predispose these patients to dysrhythmias. ${ }^{5}$ Preoperative echocardiography will help detect the presence of intracardiac shunts, ventricular hypertrophy, outlet obstruction, and valvular dysfunction.

Preoperative fasting time should be the minimum allowable fasting duration, and adequate hydration should be maintained to avoid hyperviscosity syndrome. Precaution needs to be taken to avoid any air bubbles in the IV fluid system as the patients are vulnerable to systemic air embolism. Infective endocarditis prophylaxis according to the guidelines and institutional practice should be given before the procedure.

Intraoperatively, the primary challenge is to balance the pulmonary vascular resistance (PVR) and SVR. PVR has to be kept low by avoiding hypoxia, hypercapnia, acidosis, hypothermia and by avoiding high intrathoracic peak pressures. It is better to avoid peak end-expiratory pressure, and $\mathrm{N}_{2} \mathrm{O}$ (nitrous oxide) is avoided as it increases PVR and can also worsen the air embolism if it may occur. We used fentanyl along with ketamine for induction to prevent an excessive drop in SVR during the induction phase. The drugs should be given in a titrated dose to prevent any precipitous fall in blood pressure. Anesthetic maintenance is done with sevoflurane as its effect on SVR, PVR, and the left ventricular systolic function is minimal. ${ }^{6}$ Sevoflurane also helps maintain cerebral autoregulation in concentrations up to one MAC. Euvolemic and euglycemic targets should be maintained to avoid any secondary neurological injury as well as systemic adverse effects. Hyperviscosity in already at risk brain can result in intraoperative cerebral ischemia causing neurological deficits.

Vasopressor of choice in this subset of patients would be phenylephrine as it increases the SVR without increasing the HR and also maintains the cerebral perfusion pressure. Osmotic agents such as mannitol, when administered for reducing the ICP, can produce significant shifts in the intravascular circulating volume and also worsen the RV systolic function. It is better to opt for hypertonic saline (3\%) as it produces an equiefficacious reduction in ICP with fewer volume shifts and also offer a positive inotropic effect on the myocardium.

Intraoperative use of TEE helps screen for PFO as the sensitivity of preoperative transthoracic echo is low. A mid-esophageal bicaval view will help the anesthesiologist to screen for PFO, monitor for any episodes of VAE during the neurosurgery. The mid-esophageal four/five chamber view will help in monitoring the regional and global ventricular function. TEE aids in the better management of intraoperative hypotensive episodes by identifying the causative etiology amid various causation such as VAE, hypovolemia, low RV/LV compliance, reduced SVR, and increased PVR. ${ }^{7}$ Prompt identification of the etiology will help the clinicians to take ideal corrective measures as any overzealous fluid therapy in the absence of hypovolemia will further worsen the oxygenation by increasing the extravascular lung water and also by causing hemodilution. ${ }^{1}$ It also helps monitor the shunt fraction in the presence of a VSD and helps avoid any cyanotic episodes in the intraoperative setting.

Well-tailored perioperative analgesia will help reduce the cardiac stress as pain will increase both PVR and SVR and increase the myocardial oxygen demand. At the end of the procedure, if there were no intraoperative complications and hemodynamics are stable, the patient can be assessed and extubated as in our case. Postoperatively, the patient needs vigilant monitoring to detect any worsening of cardiopulmonary status due to development of heart failure and pulmonary hypertension.

\section{Conclusion}

Challenges faced in the management of congenitally corrected TGA with VSD presenting for neurosurgery are manifold. It is beneficial to have a comprehensive knowledge of the underlying cardiac pathophysiology and its anesthetic management as this will help in understanding the need for unique monitoring modalities in the perioperative period thereby improving the patient outcome. A meticulously administered balanced general anesthesia with the goals of maintaining normal sinus rhythm, keeping PVR lower than SVR, and facilitation of lax brain is optimal.

\section{Funding}

None.

\section{Conflict of Interest}

None declared.

\section{References}

1 Graham TP, Jr, Bernard YD, Mellen BG, et al. Long-term outcome in congenitally corrected transposition of the great arteries: a multi-institutional study. J Am Coll Cardiol 2000;36(1):255-261

2 Aebi C, Kaufmann F, Schaad UB. Brain abscess in childhoodlong-term experiences. Eur J Pediatr 1991;150(4):282-286

3 Takeshita M, Kagawa M, Yonetani H, et al. Risk factors for brain abscess in patients with congenital cyanotic heart disease. Neurol Med Chir (Tokyo) 1992;32(9):667-670

4 Anderson RH, Becker AE, Arnold R, Wilkinson JL. The conducting tissues in congenitally corrected transposition. Circulation 1974;50(5):911-923

5 Fontaine JM, Kamal BM, Sokil AB, Wolf NM. Ventricular tachycardia: a life-threatening arrhythmia in a patient with congenitally corrected transposition of the great arteries. J Cardiovasc Electrophysiol 1998;9(5):517-522

6 Laird TH, Stayer SA, Rivenes SM, et al. Pulmonary-to-systemic blood flow ratio effects of sevoflurane, isoflurane, halothane, and fentanyl/midazolam with $100 \%$ oxygen in children with congenital heart disease. Anesth Analg 2002;95(5):1200-1206

7 Ayres NA, Miller-Hance W. Fyfe DA, et al; Pediatric Council of the American Society of the Echocardiography. Indications and guidelines for performance of transesophageal echocardiography in the patient with pediatric acquired or congenital heart disease: report from the task force of the Pediatric Council of the American Society of Echocardiography. J Am Soc Echocardiogr 2005;18(1):91-98 